\title{
ANÁLISE DA PRODUÇÃO CIENTÍFICA ACERCA DO CONHECIMENTO DAS MULHERES SOBRE A CONTRACEPÇÃO NO PUERPÉRIO
}

\section{ANALYSIS OF SCIENTIFIC PRODUCTION ON WOMEN'S KNOWLEDGE ABOUT CONTRACEPTION IN THE PUERPERIUM}

\section{Laís Rodrigues Silva ${ }^{1} *$ Éllen Cristina da Costa Leite Sousa ${ }^{2} *$ Mauriely Paiva de Alcântara e Silva $^{3}$ * Girzia Sammya Tajra Rocha ${ }^{4}$ Herla Maria Furtado Jorge ${ }^{5 *}$ Livia Carvalho Pereira $^{6}$}

\section{RESUMO}

Objetivo: Analisar o conhecimento que as mulheres possuem acerca da contracepção no puerpério. Metodologia: Foi realizada nas bases de dados Medline/PubMed, Web of Science, Excerpta Medica dataBase, Literatura Latino-Americana e do Caribe em Ciências da Saúde e Base de Dados de Enfermagem, considerando o período de setembro e outubro de 2020. Para análise, foramselecionados 11 artigos, 10 internacionais e um nacional. Resultados: A maioria das mulheres no período pós-parto tinham baixo conhecimento sobre os métodos contraceptivos, principalmente sobre implantes, método da lactação, amenorreia e sobre anticoncepção em mulheres soropositivas. Considerações finais:Constatou que há um déficit de conhecimento das puérperas sobre contracepção, podendo ser observado em mulheres com diversas faixas etárias, assim como, mulheres primíparas e multíparas.

Palavras-chave: Período Pós-Parto; Puerpério; Anticoncepção; Contracepção; Conhecimento.

\begin{abstract}
Objective: To analyze the knowledge that women have about contraception in the puerperium. Methodology: It was performed in the Medline/PubMed, Web of Science, Excerpta Medica dataBase, Latin American and Caribbean Literature on Health Sciences and Nursing Database databases, considering the period from September to October 2020. For analysis, 11 articles were selected, 10 international and one national. Results: Most women in the postpartum period had little knowledge about contraceptive methods, mainly about implants, lactation method, amenorrhea and about contraception in HIV-positive women. Final considerations: It was found that there is a deficit of knowledge of puerperae about contraception, and can be observed in women in variousage groups, as well as primiparous and multiparous women.
\end{abstract}

Keywords: Postpartum Period; Puerperium; Contraception; Contraception; Knowledge.

\footnotetext{
${ }^{1}$ Universidade Federal do Piauí, Teresina, Piauí, Brasil. Orcid: https://orcid.org/0000-0003-4928-8714

${ }^{2}$ Universidade Federal do Piauí, Teresina, Piauí, Brasil. Orcid: https://orcid.org/0000-0003-2561-065X

${ }^{3}$ Universidade Federal do Piauí, Teresina, Piauí, Brasil. Orcid: https://orcid.org/0000-0002-2667-1596

${ }^{4}$ Universidade Federal do Piauí, Programa de Pós-graduação em Enfermagem, Teresina, Piauí, Brasil. Orcid: https://orcid.org/0000$0002-1624-3838$

${ }^{5}$ Universidade Federal do Piauí, Programa de Pós-graduação em Enfermagem, Teresina, Piauí, Brasil. Orcid: https://orcid.org/00000001-9706-5369

${ }^{6}$ Universidade Federal do Piauí, Programa de Pós-graduação em Enfermagem, Teresina, Piauí, Brasil. Orcid: https://orcid.org/00000003-2324-107X
} 


\section{INTRODUÇÃO}

O puerpério é um período que se inicia após o parto, ou seja, após a expulsão da placenta e das membranas ovulares e onde ocorre involução do organismo da mulher após a gestação ${ }^{(1-2)}$. Quanto ao final do puerpério, estudos afirmam que pode durar até a sexta semana pós-parto ou até doze meses após o parto $^{(3)}$. No entanto, a Organização Mundial da Saúde recomenda o acompanhamento até o sexto mês puerperal.

Além do conhecimento da fisiologia do período pós-parto, as mudanças são intensas no aspecto psicológico, social, profissional, familiar e conjugal da mulher, observando a necessidade de uma rede de apoio $^{(1)}$. Ao considerar tais mudanças, observa-se que este período pode levar a agravos e consequentemente a morbidades significativas para a saúde pública, tornando um dos focos de políticas públicas voltada para a mulher em toda sua integridade ${ }^{(4)}$.

Para a garantia de uma assistência qualificada, integral, humanizada, com acesso aos direitos e aos meios de serviços e estimulação a redução de morbimortalidade feminina no Brasil, foi criado em 2004 o Programa Nacional de Atenção Integral a Saúde da Mulher (PNAISM), fundamentada nos princípios do Sistema Único de Saúde $(\mathrm{SUS})^{(5)}$. A partir da legitimação de uma política pública para as mulheres em todos os ciclos de vida, nota-se que o puerpério passou a ser incluída na atenção básica ${ }^{(6)}$.
Dentre os fatores que preocupam as autoridades públicas de saúde são: a falta de acesso à informação de pessoas desassistidas, sobre a duração da infertilidade provocada pela amamentação; os intervalos menores que dezoito meses entre duas gestações que podem levar ao aumento do risco de patologias fetal-neonatal; e o aumento da morbimortalidade feminina e aumento da recorrência de diabetes gestacional ${ }^{(7)}$. Diante disso, sugere-se intervalos maiores entre as gestações, pois evidencia-se diminuição na morbimortalidade infantil e materna, além da qualidade da saúde de ambos ${ }^{(8)}$.

Por estas razões, ações relacionadas a saúde reprodutiva assumem um papel importante para o bem-estar materno-fetal, pois os indivíduos responsáveis pela reprodução têm o direito de determinar o intervalo gestacional e evitar nova gestação não $\operatorname{programada}^{(1)}$. $\mathrm{O}$ planejamento reprodutivo ou familiar funciona como uma estratégia para auxiliar no controle da fecundidade, dentre as escolhas, os métodos contraceptivos devem ser discutidos junto ao profissional de saúde ${ }^{(5)}$.

Os métodos anticoncepcionais utilizados no pós-parto devem ser escolhidos de acordo com os critérios da Organização Mundial da saúde (OMS), onde o profissional de saúde deve realizar um acolhimento com escuta qualificada, avaliação global, orientar e escolher de acordo com a necessidade de cada paciente $^{(9)}$. Dentre os métodos, há o de 
lactação e amenorreia, métodos de barreira, dispositivos intrauterino, métodos hormonais e métodos cirúrgicos ${ }^{(10)}$.

Este estudo perpassa pela necessidade de promover ações que buscam a garantia dos direitos sexuais e reprodutivos do indivíduo, ainda pouco discutida nos serviços de saúde. Além disso, objetivou-se analisar o conhecimento das mulheres acerca da contracepção no puerpério. Nesse sentido, em face tal problemática, elaborou-se a seguinte questão norteadora: qual o conhecimento de mulheres sobre a contracepção no puerpério?

\section{METODOLOGIA}

Foi realizada uma revisão integrativa da literatura referente ao conhecimento de mulheres sobre a contracepção no puerpério. Esse tipo de estudo, possibilita a síntese do conhecimento sobre determinada temática, assim como, a inclusão de resultados significativos de estudos na prática ${ }^{(11-13)}$.

Para tal, foram realizados seis passos: 1. Identificação do tema e seleção da hipótese ou questão de pesquisa; 2. determinação de critérios para inclusão e exclusão de estudos, assim como, a busca na literatura; 3 . delimitação das informações que serão extraídas das pesquisas selecionadas; 4. categorização e avaliação dos estudos incluídos; 5. interpretação dos resultados extraídos e 6. síntese do conhecimento demonstrado $^{(14)}$.
Com o intuito de obter de modo eficiente e preciso, a melhor informação disponível, foi elaborada uma pergunta de pesquisa e utilizada para a busca bibliográfica a estratégia PICo (População, Intervenção, Controle e Desfecho/Outcome), que visa a seleção dos termos de busca, além disso, possui adequada sensibilidade para obter pesquisas do tipo qualitativas com foco nas experiências humanas e sociais. Ela contempla a população, ou o paciente ou o problema abordado (Population/Patient/ Problem) o fenomeno de (Population/Patient/Problem), o fenômeno de interesse (Interest) e o contexto (Context) ${ }^{(15)}$.

Nessa perspectiva, para realização desse estudo, P refere-se as puérperas; I a contracepção; Co ao conhecimento. Após estabelecimento das variáveis, foi formulada a seguinte pergunta norteadora: Qual o conhecimento de mulheres acerca da contracepção no puerpério? A busca bibliográfica foi realizada nas seguintes bases de dados: MEDLINE/PubMed, Web of Science, $\quad$ Excerpta $\quad$ Medica dataBASE (EMBASE) $e$ Literatura LatinoAmericana e do Caribe em Ciências da Saúde (LILACS) e Base de Dados de Enfermagem (BDENF). Após definida a estratégia de busca dos artigos, fora utilizado como critério de inclusão: estudos primários que contemplam o conhecimento de mulheres sobre a contracepção no puerpério, publicados a partir de 2010, nos idiomas português, inglês e espanhol e que possuíssem texto completo 
disponível. Fora empregado como critério de exclusão, a literatura cinzenta.

Os descritores controlados (DC) e não controlados (DNC), foram selecionados por meio da consulta dos termos no Medical Subject Headings (MeSH), por títulos extraídos da EMTREE e da biblioteca Descritores em Ciências da Saúde (DeCS), os quais serão integrados por meio dos operadores booleanos AND e OR e as palavras-chave em cada base.

A coleta de dados foi realizada entreos meses de outubro e novembro de 2020 , porduas pesquisadoras simultaneamente, e dividida em quatro etapas, na primeira etapa, os artigos levantados nas bases de dados foram submetidos a uma pré-seleção de acordo com os critérios de inclusão e exclusão anteriormente citados, examinados inicialmente através da leitura dos títulos eresumos.

Por conseguinte, os estudos selecionados foram analisados considerando o potencial de inclusão na presente revisão, avaliando-se a propriedade de responder à pergunta norteadora, o tipo de pesquisa desenvolvido, objetivos, metodologia, discussão e conclusões. Quanto a quartaetapa, foi realizada a leitura na íntegra dos artigos na definida amostragem. A última fase correspondeu à interpretação dos resultados e discussão. Das cinco bases de dados incluídas no estudo, obteve-se um total de 843 artigos. Sendo que, haviam 63 artigos duplicados, após a retirada dos repetidos, obtivemos um número de 780 artigos. Desses, 731 não se enquadravam nos critérios de inclusão e nos objetivos propostos. Após a realização da leitura dos títulos e resumos, obteve-se 49 estudos, e após a leitura dos artigos completos compuseram a amostra final 11 estudos.

As exclusões dos artigos após a leitura dos textos completos tiveram como principais justificativas o fato de não apresentarem a opinião das puérperas em relação aos métodos contraceptivos, ou ainda quando era coletado a opinião das entrevistadas não havia uma separação das puérperas com as grávidas, outras justificativas encontradas é o fato da pesquisa ter sido realizada muito tempo após o puerpério, e a ocorrência de alguns estudos realizarem uma orientação prévia sobre os métodos contraceptivos antes de avaliar o conhecimento das participantes.

A maioria dos estudos incluídos eram derivados de uma pesquisa descritiva ou qualitativa, e somente um era estudo de coorte. Os estudos de cunho descritivo ou qualitativo foram classificados com o nível de evidência 6 e o estudo de coorte teve nível de evidência 4. Posteriormente os trabalhos foram dispostos em uma tabela no Excel para apresentação dos resultados dos dados obtidos, foi realizada de forma descritiva contendo dados referentes à autoria, base onde o artigo foi encontrado, objetivo de estudo, tipo de estudo, nível de evidência e 
desfecho em relação ao conhecimento das puérperas sobre os métodos contraceptivos.

\section{RESULTADOS}

Dos onze artigos selecionados verificou-se que a maioria dos estudos foi publicado em periódicos internacionais $(n=10)$ e somente um em periódico nacional (Revista de Enfermagem da UFPE). Os artigos foram escritos em português e inglês, publicados entre 2012 e 2019, tendo um total de cinquenta e seis articulistas envolvidos nas pesquisas do estudo, variando entre um a nove autores por manuscrito. Quanto a abordagem metodológica, a maioria foi quantitativa (9) e dois estudos utilizaram a abordagem qualitativa. Observa-se que nos últimos anos a intensificação das publicações foi significativa (Quadro 1).

Quadro 1 - Caracterização dos estudos segundo título, autoria, periódico, ano de publicação, metodologia, objetivo e desfecho.

\begin{tabular}{|c|c|c|c|c|}
\hline Autoria/Título & $\begin{array}{c}\text { Periódico } \\
\text { /ano }\end{array}$ & $\begin{array}{l}\text { Tipo de } \\
\text { estudo }\end{array}$ & Objetivo & Desfecho \\
\hline $\begin{array}{l}\text { O'shea MS, Rosenberg } \\
\text { NE, Hosseinipour MC, } \\
\text { Stuart GS, Miller WC, } \\
\text { Kaliti SM, et al. }{ }^{(16)} \\
\text { Effect of HIV status on } \\
\text { fertility desire and } \\
\text { knowledge of long- } \\
\text { acting reversible } \\
\text { contraception } \\
\text { postpartum Malawian } \\
\text { women. }\end{array}$ & $\begin{array}{l}\text { AIDS } \\
\text { Care } \\
(2016)\end{array}$ & $\begin{array}{l}\text { Coorte, } \\
\text { prospectivo. }\end{array}$ & $\begin{array}{l}\text { Descrever as } \\
\text { intenções de } \\
\text { gravidez e } \\
\text { preferências de } \\
\text { planejamento } \\
\text { familiar de } \\
\text { mulheres } \\
\text { infectadas com } \\
\text { HIV e não } \\
\text { infectadas com } \\
\text { HIV pós-parto, e } \\
\text { avaliar se o } \\
\text { status de HIV } \\
\text { está associado } \\
\text { ao desejo de } \\
\text { fertilidade e ao } \\
\text { conhecimento } \\
\text { da contracepção } \\
\text { intrauterina e do }\end{array}$ & $\begin{array}{l}\text { Das } 634 \text { mulheres } \\
\text { no pós-parto, o } \\
\text { conhecimento } \\
\text { sobre os métodos } \\
\text { de contracepção } \\
\text { era baixo, a } \\
\text { maioria das } \\
\text { participantes } \\
\text { faltava algum } \\
\text { conhecimento } \\
\text { sobre a segurança } \\
\text { e eficácia da } \\
\text { contracepção } \\
\text { intrauterina em } \\
\text { relação } \\
\text { implante. As } \\
\text { mulheres a } \\
\text { infectadas pelo } \\
\text { HIV tinham }\end{array}$ \\
\hline
\end{tabular}




\begin{tabular}{|c|c|c|c|c|}
\hline & & & $\begin{array}{l}\text { implante } \\
\text { contraceptivo } \\
\text { subdérmico. }\end{array}$ & $\begin{array}{l}\text { menos } \\
\text { probabilidade de } \\
\text { saber que a } \\
\text { contracepção } \\
\text { reversível era } \\
\text { seguro para uso } \\
\text { durante } \\
\text { amamentação. }\end{array}$ \\
\hline $\begin{array}{l}\text { Lima GKS, Santos } \\
\text { AAP, Silva } \\
\text { JMO, Comassetto } \\
\text { I, Correia SR, Ferreira } \\
\text { DCS.(17) Autocuidado } \\
\text { de adolescentes no } \\
\text { período puerperal: } \\
\text { aplicação da teoria de } \\
\text { Orem. }\end{array}$ & $\begin{array}{l}\text { Revista } \\
\text { de } \\
\text { Enfermag } \\
\text { em UFPE } \\
(2017)\end{array}$ & $\begin{array}{l}\text { Estudo } \\
\text { qualitativo, } \\
\text { descritivo. }\end{array}$ & $\begin{array}{l}\text { Identificar o } \\
\text { conhecimento } \\
\text { de puérperas } \\
\text { adolescentes } \\
\text { sobre } \\
\text { autocuidado } \\
\text { baseado no } \\
\text { referencial } \\
\text { teórico } \\
\text { Dorothea } \\
\text { Elizabeth Orem. }\end{array}$ & $\begin{array}{l}\text { Sete puérperas } \\
\text { adolescentes } \\
\text { apresentaram } \\
\text { déficit no } \\
\text { autocuidado por } \\
\text { meio do } \\
\text { conhecimento } \\
\text { negativo sobre } \\
\text { anticoncepção e } \\
\text { os benefícios da } \\
\text { amamentação; }\end{array}$ \\
\hline $\begin{array}{lr}\text { Potgieter } F, & \text { Kapp } P \text {, } \\
\text { Coetzee } \text { F. }^{(18)} & \text { Factors } \\
\text { influencing } & \text { post- } \\
\text { partum } & \text { women's } \\
\text { choice of } & \text { an } \\
\text { implantable } & \\
\text { contraceptive device in } \\
\text { a rural district hospital } \\
\text { in South Africa. }\end{array}$ & $\begin{array}{l}\text { South } \\
\text { African } \\
\text { Family } \\
\text { Practice } \\
(2018)\end{array}$ & $\begin{array}{l}\text { Estudo } \\
\text { qualitativo }\end{array}$ & $\begin{array}{l}\text { Explorar os } \\
\text { fatores que } \\
\text { influenciam a } \\
\text { escolha de um } \\
\text { implante } \\
\text { anticoncepcional } \\
\text { subdérmico } \\
\text { pelas mulheres. }\end{array}$ & $\begin{array}{l}\text { Entrevistas com } \\
\text { dez participantes, } \\
\text { onde } \\
\text { conhecimento } \\
\text { sobre os métodos } \\
\text { contraceptivos } \\
\text { disponíveis entre } \\
\text { as participantes } \\
\text { do pós-parto } \\
\text { permanecia } \\
\text { insuficiente. }\end{array}$ \\
\hline
\end{tabular}




\begin{tabular}{|c|c|c|c|c|}
\hline $\begin{array}{l}\text { Patel R, Panchal P, } \\
\text { Mehta A, Shah S. }{ }^{(19)} \\
\text { A knowledge, attitude } \\
\text { and practice study } \\
\text { of contraception amon } \\
\text { gst immediate post- } \\
\text { partum patients in a } \\
\text { tertiary care institute } \\
2016 .\end{array}$ & $\begin{array}{l}\text { Pesquisa } \\
\text { e prática } \\
\text { de } \\
\text { medicina } \\
\text { atual } \\
\text { (2017). }\end{array}$ & $\begin{array}{l}\text { Transversal } \\
\text { quantitativo }\end{array}$ & $\begin{array}{l}\text { Avaliar } \mathrm{o} \\
\text { conhecimento e } \\
\text { ver a atitude e } \\
\text { prática } \\
\text { do uso de contra } \\
\underline{\text { cepção entre }} \\
\text { pacientes no } \\
\text { pós-parto } \\
\text { imediato. }\end{array}$ & $\begin{array}{l}\text { A maioria dos } \\
\text { entrevistados } \\
\text { sabia } \\
\text { sobre anticoncepc } \\
\text { ionais } \\
\text { de barreira - } \\
\text { preservativos ma } \\
\text { sculinos (91,3\%), } \\
\text { seguido de pílulas } \\
\text { hormonais } \\
\text { (72,2\%), } \\
\text { (64,3\%) e DMU } \\
\text { (11,3\%). Apenas } \\
\text { 20\% conheciam a } \\
\text { anticoncepção de } \\
\text { emergência. }\end{array}$ \\
\hline 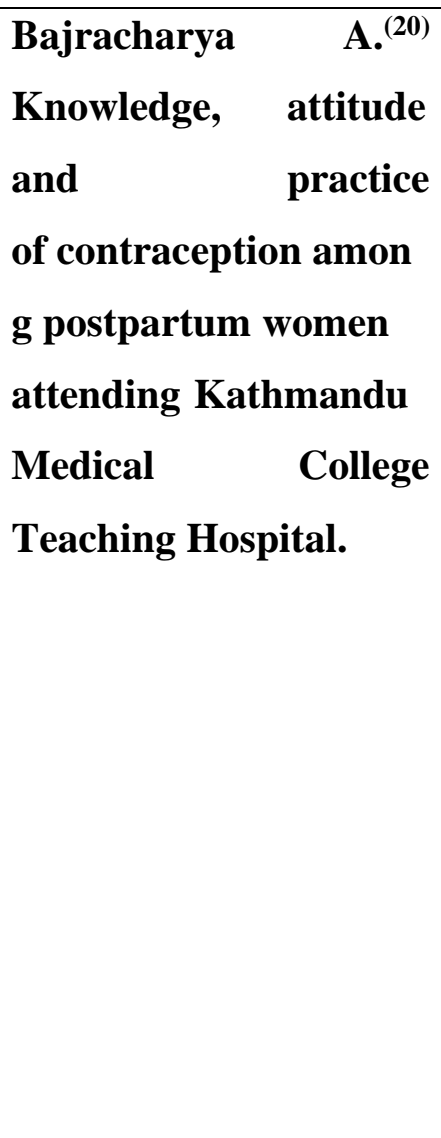 & $\begin{array}{l}\text { Kathman } \\
d u \\
\text { Universit } \\
\text { y Medical } \\
\text { Journal } \\
\text { (2015) }\end{array}$ & $\begin{array}{l}\text { Descritivo, } \\
\text { quantitativo }\end{array}$ & $\begin{array}{l}\text { Determinar o } \\
\text { conhecimento, a } \\
\text { atitude e a } \\
\text { prática de } \\
\text { contracepção } \\
\text { entre puérperas } \\
\text { atendidas em um } \\
\text { hospital escola. }\end{array}$ & $\begin{array}{l}\text { Das } 400 \text { mulheres } \\
\text { entrevistadas, a } \\
\text { maioria }(90,8 \%) \\
\text { tinha } \\
\text { conhecimento do } \\
\text { uso de } \\
\text { anticoncepcionais } \\
\text { Entre 60,5\% das } \\
\text { mulheres que já } \\
\text { haviam feito uso } \\
\text { de } \\
\text { anticoncepcionais } \\
\text { anticoncepcionais } \\
\text { orais foram os } \\
\text { mais comuns. }\end{array}$ \\
\hline
\end{tabular}




\begin{tabular}{|c|c|c|c|c|}
\hline $\begin{array}{l}\text { Özsoy S, Aksu } \\
\text { H, Balkaya NA, Horta } \\
\text { GD. }{ }^{(21)} \text { Knowledge and } \\
\text { Opinions } \\
\text { of Postpartum Mothers } \\
\text { About the Lactational } \\
\text { Amenorrhea Method: } \\
\text { The Turkish } \\
\text { Experience. }\end{array}$ & $\begin{array}{l}\text { Journal of } \\
\text { the } \\
\text { Academy } \\
\text { of Breastf } \\
\text { eeding } \\
\text { Medicine } \\
\text { (2018) }\end{array}$ & $\begin{array}{l}\text { Descritivo, } \\
\text { quantitativo }\end{array}$ & $\begin{array}{l}\text { Investigar a } \\
\text { opinião e o } \\
\text { conhecimento } \\
\text { das mães sobre } \\
\text { as características } \\
\text { contraceptivas } \\
\text { da } \\
\text { amamentação. }\end{array}$ & $\begin{array}{l}\text { Das } 400 \\
\text { puérperas, } 38,9 \% \\
\text { das mães tem } \\
\text { conhecimento } \\
\text { sobre o efeito } \\
\text { anticoncepcional } \\
\text { da amamentação. }\end{array}$ \\
\hline $\begin{array}{l}\text { Rossman B, Asiodu I, } \\
\text { Hoban R, Patel AL, } \\
\text { Engstrom JL, Medina- } \\
\text { Poeliniz C, et al. }{ }^{(22)} \\
\text { Priorities } \\
\text { for Contraception and } \\
\text { Lactation Among } \\
\text { Breast Pump- } \\
\text { Dependent Mothers of } \\
\text { Premature Infants in } \\
\text { the Neonatal Intensive } \\
\text { Care Unit. }\end{array}$ & $\begin{array}{l}\text { Journal of } \\
\text { the } \\
\text { Academy } \\
\text { of Breastf } \\
\text { eeding } \\
\text { Medicine } \\
\text { (2019). }\end{array}$ & $\begin{array}{l}\text { Descritivo, } \\
\text { quantitativo }\end{array}$ & $\begin{array}{l}\text { Determinar o } \\
\text { conhecimento e } \\
\text { as prioridades } \\
\text { para a } \\
\text { contracepção } \\
\text { pós-parto e a } \\
\text { lactação em } \\
\text { mães de bebês } \\
\text { prematuros. }\end{array}$ & $\begin{array}{l}\text { Das } 25 \text { mães } \\
\text { prematuras } \\
\text { entrevistadas, } \\
60 \% \text { das mães } \\
\text { planejavam usar } \\
\text { anticoncepcionais } \\
\text { tiveram dúvidas } \\
\text { quanto à forma } \\
\text { correta } \\
\text { consumo. }\end{array}$ \\
\hline $\begin{array}{l}\text { Giacomo PD, Sbarlati } \\
\text { A, Bagnasco A, Sasso } \\
\text { L. }{ }^{(23)} \\
\text { Woman's contraceptiv } \\
\text { e needs and } \\
\text { preferences in } \\
\text { the postpartum period: } \\
\text { an Italian study. }\end{array}$ & $\begin{array}{l}\text { Revista } \\
\text { de } \\
\text { Enfermag } \\
\text { em } \\
\text { Clínica } \\
\text { (2013) }\end{array}$ & $\begin{array}{l}\text { Descritivo, } \\
\text { quantitativo }\end{array}$ & $\begin{array}{l}\text { Descrever o que } \\
\text { as puérperas } \\
\text { sabem sobre a } \\
\text { contracepção } \\
\text { pós-parto e } \\
\text { identificar suas } \\
\text { necessidades e } \\
\text { expectativas. }\end{array}$ & $\begin{array}{l}\text { Das } 300 \text { mulheres } \\
\text { puérperas, } \\
\text { durante } \\
\text { gravidez e o pós- } \\
\text { parto, 45,5\% das } \\
\text { mulheres } \\
\text { relataram ter } \\
\text { recebido } \\
\text { informações } \\
\text { adequadas sobre } \\
\text { contracepção. }\end{array}$ \\
\hline
\end{tabular}




\begin{tabular}{|c|c|c|c|c|}
\hline $\begin{array}{l}\text { Lathrop E, Telemaque } \\
\text { Y, Haddad L, } \\
\text { Stephenson } \\
\text { Goedken P, Cwiak C, } \\
\text { et }{ }^{(24)} \\
\text { Knowledge and use of } \\
\text { and opportunities for } \\
\text { emergency contracepti } \\
\text { on in Northern Haiti. }\end{array}$ & $\begin{array}{l}\text { Revista } \\
\text { Internacio } \\
\text { nal de } \\
\text { Ginecolo } \\
\text { gia e } \\
\text { Obstetríci } \\
\text { a (2013) }\end{array}$ & $\begin{array}{l}\text { Descritivo, } \\
\text { quantitativo }\end{array}$ & $\begin{array}{l}\text { Avaliar o } \\
\text { conhecimento, a } \\
\text { experiência e o } \\
\text { desejo da } \\
\text { anticoncepção } \\
\text { de emergência } \\
\text { (CE) no } \\
\text { mulheres no } \\
\text { pós-parto nat. } \\
\text { Haiti. }\end{array}$ & $\begin{array}{l}\text { Das } \\
\text { mulheres, } \\
(58,2 \%) \\
\text { conheciam o } \\
\text { conceito de AE } \\
\text { como medida de } \\
\text { emergência no } \\
\text { período pós- } \\
\text { coito. Destes, } 130 \\
(89,7 \%) \\
\text { conheciam apenas } \\
\text { métodos } \\
\text { tradicionais. }\end{array}$ \\
\hline $\begin{array}{l}\text { Shabiby MM, Karanja } \\
\text { JG, Odawa F, Kosgei } \\
\text { R, Kibore MW, Kiarie } \\
\text { JN, et al. }^{(25)} \\
\text { Factors influencing } \\
\text { uptake } \\
\text { of contraceptive impla } \\
\text { nts in the } \\
\text { immediate postpartum } \\
\text { period among HIV } \\
\text { infected and } \\
\text { uninfected women at } \\
\text { two Kenyan District } \\
\text { Hospitals. }\end{array}$ & $\begin{array}{l}\text { Revista } \\
\text { Saúde do } \\
\text { Homem } \\
\text { BMC } \\
(2015)\end{array}$ & $\begin{array}{l}\text { Transversal } \\
\text { quantitativa }\end{array}$ & $\begin{array}{l}\text { Determinar a } \\
\text { aceitação e os } \\
\text { fatores que } \\
\text { influenciam a } \\
\text { ingestão de } \\
\text { implantes } \\
\text { anticoncepcionai } \\
\text { s pós-parto } \\
\text { imediato entre } \\
\text { mulheres } \\
\text { infectadas com } \\
\text { HIV e não } \\
\text { infectadas. }\end{array}$ & $\begin{array}{l}\text { Das } \\
\text { entrevistadas, a } \\
\text { maioria estava } \\
\text { ciente dos } \\
\text { métodos } \\
\text { modernos com } \\
\text { anticoncepcionais } \\
\text { injetáveis (93\%) } \\
\text { pílulas } \\
\text { anticoncepcionais } \\
\text { (92\%). Métodos } \\
\text { naturais como } \\
\text { amenorréia } \\
\text { lactacional } \\
\text { (LAM) (3\%) e } \\
\text { métodos de ritmo } \\
(2,7 \%) \text { estavam } \\
\text { entre os métodos } \\
\text { menos } \\
\text { conhecidos. }\end{array}$ \\
\hline
\end{tabular}




\begin{tabular}{|c|c|c|c|c|}
\hline $\begin{array}{l}\text { Credé S, Hoke T, } \\
\text { Constant D, Green MS, } \\
\text { Moodley J, Harries } \\
\text { J. }{ }^{(26)} \text { Factors } \\
\text { impacting knowledge a } \\
\text { nd use of long acting } \\
\text { and } \\
\text { permanent contracepti } \\
\text { ve methods by postpart } \\
\text { um HIV positive and } \\
\text { negative women in } \\
\text { Cape Town, South } \\
\text { Africa: a cross- } \\
\text { sectional study. }\end{array}$ & $\begin{array}{l}\text { Revista } \\
\text { de Saúde } \\
\text { Pública } \\
\text { BMC } \\
(2012)\end{array}$ & $\begin{array}{l}\text { Transversal } \\
\text { quantitativa. }\end{array}$ & $\begin{array}{l}\text { Comparar os } \\
\text { fatores que } \\
\text { influenciam a } \\
\text { escolha em } \\
\text { contracepção e } \\
\text { no } \\
\text { conhecimento e } \\
\text { atitudes das } \\
\text { mulheres em } \\
\text { relação ao DIU } \\
\text { e à esterilização } \\
\text { feminina pelo } \\
\text { status de HIV. }\end{array}$ & $\begin{array}{l}\text { Foi entrevistado } \\
265 \text { mulheres } \\
\text { HIV positivas e } \\
273 \text { mulheres } \\
\text { pós-parto HIV } \\
\text { negativas. } \\
\text { Verificou-se que } \\
\text { o conhecimento } \\
\text { insuficiente sobre } \\
\text { os métodos de } \\
\text { longa duração e } \\
\text { permanentes } \\
\text { provavelmente } \\
\text { está contribuindo } \\
\text { para a má } \\
\text { aceitação desses } \\
\text { métodos. }\end{array}$ \\
\hline
\end{tabular}

Fonte: Os autores.

Constatou-se que os objetivos dos estudos variaram desde o interesse em avaliar o conhecimento das mulheres no pós-parto sobre a contracepção, descrever as características contraceptivas da amamentação e descrever o conhecimento e aceitação das mães soropositivas sobre os principais métodos anticoncepcionais. Em relação ao desfecho de cada artigo, o tamanho das amostras variou entre 7 e 634 mulheres, sendo adolescentes puérperas e puérperas.
Identificou-se nos artigos o conhecimento das mulheres sobre o uso de métodos contraceptivos durante o puerpério em seu contexto de vida e a influência desse conhecimento para a escolha do método. Portando, a síntese apresentada no Quadro 1 dos manuscritos apresentados mostra três principais temas abordados possíveis de análise, onde foram agrupados de acordo a respectiva categoria (Quadro 2).

Quadro 2 - Síntese do conhecimento de acordo com as categorias temáticas.

\begin{tabular}{|l|l|l|}
\hline $\begin{array}{l}\text { Tipos de contracepçãono } \\
\text { puerpério }\end{array}$ & Estudo & Resultados/Conclusões \\
\hline
\end{tabular}




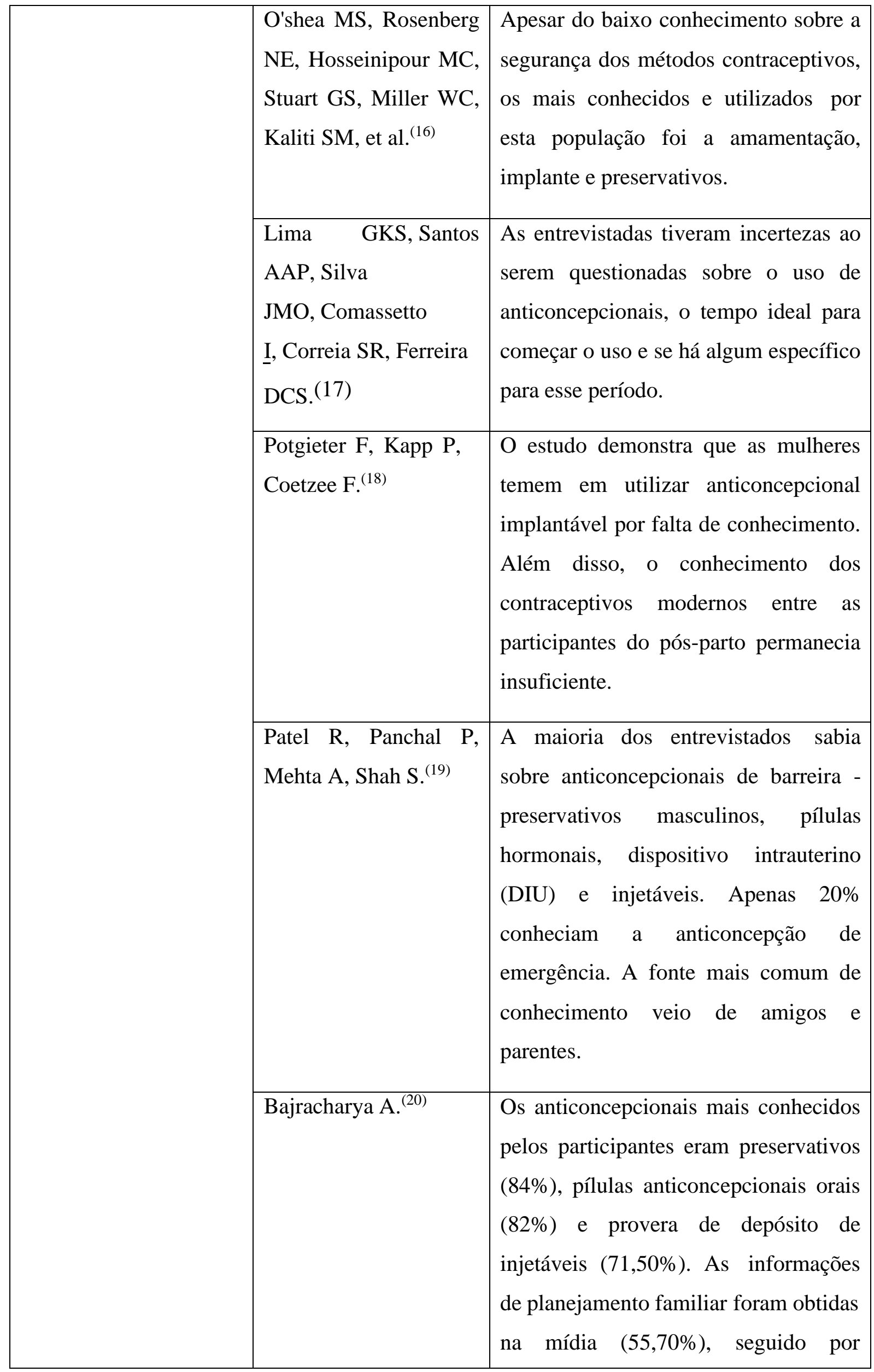




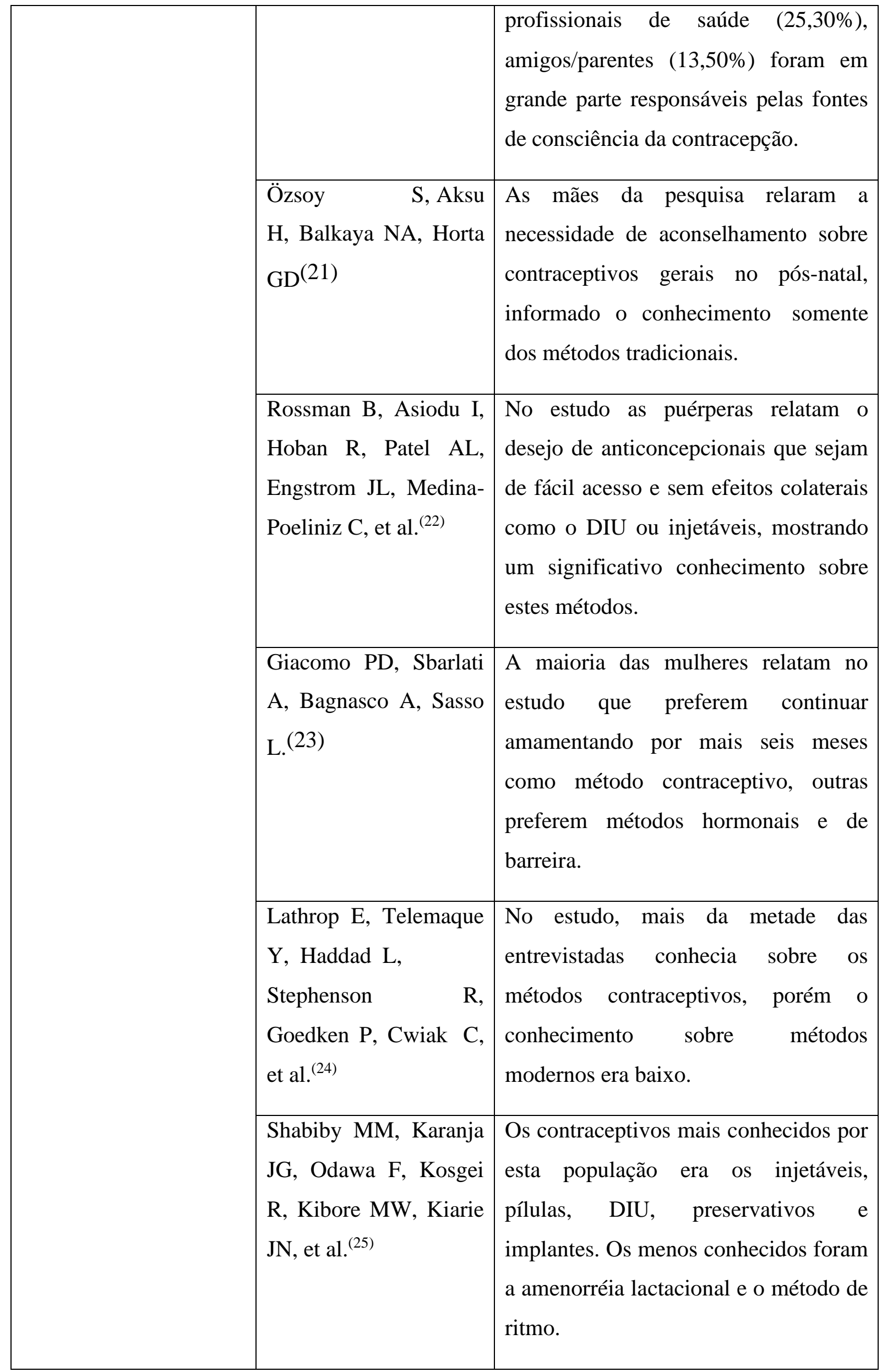




\begin{tabular}{|c|c|c|}
\hline \multirow[t]{3}{*}{$\begin{array}{l}\text { Benefícios da } \\
\text { amamentação }\end{array}$} & $\begin{array}{l}\text { Lima GKS, Santos } \\
\text { AAP, Silva } \\
\text { JMO, Comassetto } \\
\text { I, Correia SR, Ferreira } \\
\text { DCS. (17) }\end{array}$ & $\begin{array}{l}\text { Observou-se lacunas no conhecimento } \\
\text { das puérperas adolescentes sobre os } \\
\text { benefícios da amamentação para a } \\
\text { saúde materno-infantil que, segundo a } \\
\text { análise de seus depoimentos, foram } \\
\text { decorrentes da carência de instrução } \\
\text { sobre esses aspectos. }\end{array}$ \\
\hline & $\begin{array}{l}\text { Özsoy S, Aksu } \\
\text { H, Balkaya NA, Horta } \\
\text { GD. }{ }^{(21)}\end{array}$ & $\begin{array}{l}\text { Grande parte das mulheres não tinham } \\
\text { conhecimento sobre o método de } \\
\text { lactação e amenorreia e sua eficácia. }\end{array}$ \\
\hline & $\begin{array}{l}\text { Rossman B, Asiodu I, } \\
\text { Hoban R, Patel AL, } \\
\text { Engstrom JL, Medina- } \\
\text { Poeliniz C, et al. }\end{array}$ & $\begin{array}{l}\text { As puérperas de prematuros mostram } \\
\text { interesse em conhecer métodos } \\
\text { eficazes que priorize o bebê } \\
\text { dependentes de bomba de leite e assim } \\
\text { ir em busca de outros métodos que não } \\
\text { seja de lactação e amenorreia. }\end{array}$ \\
\hline \multirow[t]{2}{*}{$\begin{array}{l}\text { Anticoncepção em } \\
\text { mulheres com HIV }\end{array}$} & $\begin{array}{l}\text { O'shea MS, Rosenberg } \\
\text { NE, Hosseinipour MC, } \\
\text { Stuart GS, Miller WC, } \\
\text { Kaliti SM, et al. }{ }^{(16)}\end{array}$ & $\begin{array}{l}\text { As puérperas infectadas com HIV } \\
\text { obtêm pouco conhecimento sobre } \\
\text { contracepção reversível de ação } \\
\text { prolongada. Evidente a necessidade de } \\
\text { melhorar o acesso e o conhecimento } \\
\text { das formas de anticoncepção para } \\
\text { atender adequadamente essa } \\
\text { população. }\end{array}$ \\
\hline & $\begin{array}{l}\text { Shabiby MM, Karanja } \\
\text { JG, Odawa F, Kosgei } \\
\text { R, Kibore MW, Kiarie } \\
\text { JN, et al. }\end{array}$ & $\begin{array}{l}\text { Dentre todos os métodos, a } \\
\text { esterilização era mais conhecida pelas } \\
\text { mulheres com HIV. } \\
\text { Observa-se que é necessário } \\
\text { desenvolver estratégias para melhorar } \\
\text { o acesso e participação dos parceiros e } \\
\text { alerta sobre os efeitos colaterais dos } \\
\text { contraceptivos nesta população. }\end{array}$ \\
\hline
\end{tabular}




\begin{tabular}{|l|l|l|}
\hline & $\begin{array}{l}\text { Credé S, Hoke T, } \\
\text { Constant D, Green MS, estudo mostrou a falta de } \\
\text { Moodley J, Harries } \\
\text { J.(26) }\end{array}$ & $\begin{array}{l}\text { conhecimento de grande parte das } \\
\text { mulheres com HIV sobre a escolha do } \\
\text { método. }\end{array}$ \\
\hline
\end{tabular}

Fonte: Os autores.

\section{DISCUSSÃO}

A partir da síntese dos artigos incluídos na revisão integrativa, analisou-se que a maioria dos estudos abordaram temas significativos para discussão, como os conhecimentos das mulheres em diferentes ciclos de vida sobre os métodos contraceptivos durante o pós-parto, o conhecimento sobre o benefício da amamentação durante este período e também sobre a anticoncepção em mulheres soropositivas.

Evidenciou-se que a maioria das mulheres que participaram das pesquisas tem baixo conhecimento sobre os métodos anticoncepcionais no período puerperal e esse conhecimento se restringe aos métodos tradicionais como preservativos, pílulas anticoncepcionais orais e injetáveis ${ }^{(16-17,19-20)}$. Do mesmo modo, uma pesquisa realizada na África do Sul mostrou que as puérperas entrevistadas não utilizavam métodos modernos, como implantes, por medo devido à falta de conhecimento ${ }^{(18)}$.

$\operatorname{Rossman}^{(22)}$ sinaliza que $60 \%$ das mulheres entrevistadas sentem o desejo de ter fácil acesso e conhecer métodos contraceptivos que tenham menos efeitos colaterais, porém apresentam dúvidas quanto à forma correta do uso. Isso mostra que estas mães tem necessidades de orientação e encorajamento dos profissionais de saúde para o uso de métodos como DIU e injetáveis.

Quanto ao conhecimento de puérperas adolescentes baseada na teoria de Dorothea Orem, verificou que a maior parte das participantes apresentaram déficit no autocuidado relacionado a falta de conhecimento dos anticoncepcionais ${ }^{(17)}$. Percebeu-se a existência de fatores que contribuem para este déficit, como a falta de orientação sexual, baixa escolaridade, uso inadequado de anticoncepcionais e aconselhamento ineficaz ${ }^{(27-28)}$.

Outro ponto importante foi o conhecimento das mulheres acerca da eficácia do método da lactação e amenorreia (LAM), onde há um efeito inibidor da fertilidade em mulheres que amamentam nos primeiros seis meses pós-parto de forma exclusiva com amenorreia $^{(29)}$. Lima ${ }^{(17)}, \quad$ Ozsoy $^{(21)}$ e Rossman $^{(22)}$ por sua vez, demonstram em seus estudos que há lacunas no conhecimento da eficácia da amamentação como contraceptivo e que as puérperas mostram interesse em conhecer outros métodos que não interfiram na alimentação de seus bebês. 
Em relação ao conhecimento de puérperas soropositivas sobre contracepção, estudos de revisão constataram que há pouco conhecimento na escolha do método e anseio por interações medicamentosas devido $\mathrm{o}$ tratamento antirretroviral feita por estas mulheres ${ }^{(16,25-26)}$. Do mesmo modo, uma pesquisa realizada em Fortaleza com mulheres em uma maternidade mostrou que apenas $19,4 \%$ tinham conhecimento adequado sobre o tema ${ }^{(30)}$.

Diante da análise sobre a temática, a atenção em anticoncepção deve ser oferecida a todas as pessoas por ser um direito sexual, onde possam ter a possibilidade de escolher a melhor contracepção de acordo com a etapa reprodutiva $^{(29)}$. Além disso, o profissional de saúde assume um papel crucial para a garantia deste direito por meio de aconselhamento e oferta de informações acerca do assunto para esta população.

\section{CONSIDERAÇÕES FINAIS}

Concluiu-se que ainda há um déficit de conhecimento das puérperas sobre contracepção, podendo ser observado em mulheres com diversas faixas etárias, assim como, mulheres primíparas e multíparas. Dessa forma, se faz necessário, o aconselhamento da mulher durante o pré-natal sobre o planejamento familiar, e que se possível, que ela decida qual método irá usar após a gravidez, assim como, o planejamento e a educação em saúde devam continuar no puerpério pois algumas mulheres mudam o método de contracepção após o puerpério.

As mulheres que possuem algum conhecimento na maioria das vezes não conhecem os contraceptivos modernos, e a compreensão que elas possuem sobre algum método é bem superficial, desconhecendo seus efeitos adversos e em alguns casos, não sabiam o modo correto de utilizar, comprometendo a eficácia do método, ou ainda no caso de não ter informações suficientes, pode causar estranheza para ela, não querendo usar o método ou ainda usando da forma errada.

Entre as limitações, o fato de que na maioria dos estudos realizados os pesquisadores interferiam no conhecimento das puérperas, pois lhe davam informações sobre os métodos contraceptivos, ou ainda não avaliavam o conhecimento das puérperas, outros estudos juntaram um grupo de mulheres pra realizar o estudo sem distinguir as gestantes das puérperas, esses fatores fizeram com que a amostra diminuísse bastante.

\section{REFERÊNCIAS}

1. Stanton TA, Blumenthal PD. Postpartum hormonal contraception in breastfeeding women. Current Opinion in Obstetrics and Gynecology. 2019; 31(6):441-446.

DOI: 10.1097/GCO.0000000000000571

2. Andrade RD, Santos JS, Maia MAC, Mello DFD. Factors related to women's health in puerperium and repercussions on child health. 
Esc. Anna Nery. 2015;19(1):181-186. DOI: https://doi.org/10.5935/1414-8145.20150025

3. Baratieri $T$, Natal $S$, Hartz ZMA. Postpartum care for women in primary care: building an assessment model. Cad. Saúde Pública. 2020;36(7):1-14. DOI: https://doi.org/10.1590/0102-311X00087319

4. Ayers S, Crawley R, Button S, Thornton A, Field AP, Flood C, et al. Evaluation of expressive writing for postpartum health: a randomised controlled trial. J Behav Med. 2018;41(5):614-26.

DOI:

https://doi.org/10.1007/s10865-018-9970-3.

5. Rodrigues ESRC, Torquato JA, Davim RMB, Oliveira LFM, Alves ESRC, Nóbrega MF. Perception of women on their rights in pregnancy and childbirth. Rev enferm UFPE. 2016; 10(5): 1796-1804. DOI: 10.5205/reuol.9003-78704-1-SM.1005201628

6. Baratieri T, Natal S. Postpartum program actions in primary health care: an integrative review. Ciênc Saúde Colet. 2019;24(11):42274238. DOI: https://doi.org/10.1590/1413812320182411.28112017

7. Vieira CS, Brito MB, Yazlle MEHD. Postpartum contraception. Rev Bras Ginecol Obstet. 2008;30(9):470-479. DOI: http://dx.doi.org/10.1590/S0100-

72032008000900008

8. Silva BGCD, Lima NP, Silva SGD, Antúnez SF, Seerig LM, Restrepo-Méndez MC, et al. Maternal mortality in Brazil from 2001 to 2012: time trends and regional differences. Rev Bras Epidemiol. 2016;19(3):484-493. DOI: https://doi.org/10.1590/1980-

5497201600030002

9. Ferreira LF, D'avila AMFC, Safatle GCB. The use of contraceptive pill and alterations of main metabolic pathways. FEMINA. 2019;47(7):426-432, 2019.

Disponível em: http://docs.bvsalud.org/biblioref/2019/12/10465 33/femina-2019-477-426-432.pdf

10. Alves MDO, Parreira BDM, Dias FA, Mendes LC, Elias TC, Riul SDS. The use of contraceptive methods and factors related to the pregnancy planning among mothers. Enferm UFSM. 2016;6(3):424-433. DOI: https://doi.org/10.5902/2179769221249

11. Whittemore R, Knal K. The integrative review: updated methodology. J Adv Nurs. 2005;52(5):546-553.

DOI: https://doi.org/10.1111/j.1365-

2648.2005.03621.x

12. Mendes KDS, Silveira RCCP, Galvão CM. Integrative literature review: a research method to incorporate evidence in health care and nursing. Texto Contexto Enferm. 2008;17(4):758-764. DOI: https://doi.org/10.1590/S010407072008000400018

13. Ercole FF, Melo LS, Alcoforado CLGC. Integrative review versus systematic review. rev. min. enferm. 2014;18(1):9-12. DOI: http://www.dx.doi.org/10.5935/14152762.20140001

14. Souza MT, Silva MD, Carvalho R. Integrative review: what is it? How to do it? Einstein (São Paulo). 2010;8(1):102-106. Disponível em: https://www.scielo.br/pdf/eins/v8n1/pt_16794508-eins-8-1-0102

15. Stern C, Jordan Z, Mcarthur A. Developing the review question and inclusion criteria: The first steps in conducting a systematic review. Am. j. nurs. 2014;114(4):53-56. DOI: 10.1097/01.NAJ.0000445689.67800.86

16. O'shea MS, Rosenberg NE, Hosseinipour MC, Stuart GS, Miller WC, Kaliti SM, et al. Effect of HIV status on fertility desire and knowledge of long-acting reversible contraception of postpartum Malawian women. AIDS Care. 2015;27(4):489-498. DOI: $10.1080 / 09540121.2014 .972323$

17. Lima GKS, Santos AAP, Silva JMO, Comassetto I, Correia SR, Ferreira DCS. Self-care of adolescents in the puerperal period: application of the orem theory. Rev. enferm. UFPE on line. 2017;11(10):4217-4225. DOI: 10.5205/reuol.10712-95194-3-

SM.1110sup201727 
18. Potgieter F, Kapp P, Coetzee F. Factors influencing post-partum women's choice of an implantable contraceptive device in a rural district hospital in South Africa. S Afr Fam Pract. 2018;60(6):174-180. DOI: 10.4102/safp.v60i6.5011

19. Patel R, Panchal P, Mehta A, Shah S. A knowledge, attitude and practice study of contraception amongst immediate postpartum patients in a tertiary care institute 2016. Curr Med Res Pract. 2017;7(4):128-131. DOI: https://doi.org/10.1016/j.cmrp.2017.06.003

20. Bajracharya A. Knowledge, attitude and practice of contraception among postpartum women attending Kathmandu Medical College Teaching Hospital. Kathmandu Univ Med J. 2015;52(13):292-297.

DOI:

https://doi.org/10.3126/kumj.v13i4.16826

21. Özsoy S, Aksu H, Balkaya NA, Horta GD. Knowledge and Opinions of Postpartum Mothers About the Lactational Amenorrhea Method: The Turkish Experience. Breastfeed Med. 2018;13(1):70-74. DOI: https://doi.org/10.1089/bfm.2017.0046

22. Rossman B, Asiodu I, Hoban R, Patel AL, Engstrom JL, Medina-Poeliniz C, et al. Priorities for Contraception and Lactation Among Breast Pump-Dependent Mothers of Premature Infants in the Neonatal Intensive Care Unit. Breastfeed Med. 2019;17(7):448-455. DOI: https://doi.org/10.1089/bfm.2019.0007

23. Giacomo PD, Sbarlati A, Bagnasco A, Sasso L. Woman's contraceptive needs and preferences in the postpartum period: an Italian study. J. clin. nurs. 2013;22(1):3406-3417. DOI: https://doi.org/10.1111/jocn.12432

24. Lathrop E, Telemaque $\mathrm{Y}$, Haddad L, Stephenson R, Goedken P, Cwiak C, et al. Knowledge and use of and opportunities for emergency contraception in Northern Haiti. Int J Gynaecol Obstet. 2013;121(1):60-63. DOI: https://doi.org/10.1016/j.ijgo.2012.11.013

25. Shabiby MM, Karanja JG, Odawa F, Kosgei R, Kibore MW, Kiarie JN, et al. Factors influencing uptake of contraceptive implants in the immediate postpartum period among HIV infected and uninfected women at two Kenyan District Hospitals. BMC womens health. 2015;15(1):1-8. DOI: https://doi.org/10.1186/s12905-015-0222-1

26. Credé S, Hoke T, Constant D, Green MS, Moodley J, Harries J. Factors impacting knowledge and use of long acting and permanent contraceptive methods by postpartum HIV positive and negative women in Cape Town, South Africa: a cross-sectional study. BMC public health. 2012;12(197): 1-9. DOI: https://doi.org/10.1186/1471-2458-12-197

27. Araújo AKL, Araujo FACA, Araújo TME, Nery IS, Rocha SS. Contraception in adolescence: knowledge, chosen methods and criteria adopted. Rev. Pesqui. (Univ. Fed. Estado Rio J., Online). 2015;7(3):2815-2825. DOI: $10.9789 / 2175-5361$

28. Borovac-Pinheiro A, Jesus EAR, Surita FG. Empowering Adolescent Mothers in the Choice of Contraceptive Methods at the Postpartum Period: Avoiding a Subsequent Pregnancy. Rev. Bras. Ginecol. Obstet. 2019;41(10):607-612. DOI: http://dx.doi.org/10.1055/s-0039-1697985

29. Justino GBS, Soares GCF, Baraldi NGT, Iraí MC, Salim NR. Sexual and reproductive health in the puerperium: women's experiences. Rev enferm UFPE. 2019; 13: 1-10. DOI: https://doi.org/10.5205/1981-8963.2019.240054

30. Brasil RFG, Moreira MMC, Teles LMR, Damasceno AKDC, Moura ERF. Level of knowledge, attitudes and practices of puerperal women on HIV infection and its prevention. Acta paul. Enferm. 2014;27(2):133-137. DOI: http://dx.doi.org/10.1590/1982-0194201400024

\section{Autor correspondente}

Laís Rodrigues Silva, Endereço: Rua Belém, $\mathrm{n}^{\circ}$ 2390, Bairro: Três Andares Tel: (86) 988864038 , e-mail: laisilva1251@gmail.com.

Submissão: 2021-06-17

Aprovado: 2021-08-25 